completed CDAT and Biagi questionnaires, with at least 4 duodenal biopsies taken from D2 in addition to at least one biopsy from the duodenal bulb. The presence (Marsh 3a or above) or absence (Marsh 0-II) of villous atrophy was used to determine the sensitivities of the tests.

Results 151 patients were recruited, 101 females (66.9\%), median age 55.0 years, median duration of GFD of 72.0 months. Table 1 outlines the sensitivity and specificity of the CDAT questionnaire, Biagi questionnaire, IgA-TTG and IgAEMA.

Conclusions The sensitivity of the CDAT questionnaire was not superior to IgA-TTG for predicting villous atrophy in patients with coeliac disease. However, the use of a combination of both Biagi and CDAT had a greater sensitivity than IgA-TTG and IgA-EMA $(\mathrm{p}<0.05)$, but lower specificity $(\mathrm{p}<0.05)$. Duodenal biopsy remains the gold standard, although these scores remain useful tools in the assessment of dietary adherence.

\begin{tabular}{|c|c|c|c|c|}
\hline & $\begin{array}{l}\text { Sensitivity } \\
\%(\mathrm{Cl})\end{array}$ & $\begin{array}{l}\text { Specificity } \\
\%(\mathrm{Cl})\end{array}$ & $\begin{array}{l}\text { Positive predictive } \\
\text { value } \%(\mathrm{Cl})\end{array}$ & $\begin{array}{l}\text { Negative predictive } \\
\text { value } \%(\mathrm{Cl})\end{array}$ \\
\hline CDAT & $\begin{array}{l}52.0(37.6- \\
66.1)\end{array}$ & $\begin{array}{l}69.8(60 .- \\
7.5)\end{array}$ & $40.6(28.8-53.6)$ & $78.6(69.6-85.5)$ \\
\hline Biagi & $\begin{array}{l}22.4 \\
(12 .-7.0)\end{array}$ & $\begin{array}{l}93.1(85.6- \\
97.0)\end{array}$ & $\begin{array}{l}61.1 \\
(36.1-81.7)\end{array}$ & $\begin{array}{l}71.4 \\
(62.8-78.8)\end{array}$ \\
\hline $\begin{array}{l}\text { CDAT \& } \\
\text { Biagi }\end{array}$ & $\begin{array}{l}61.2(46 .- \\
4.8)\end{array}$ & $\begin{array}{l}69.3(60 .- \\
7.2)\end{array}$ & $43.5(35 .-2.0)$ & $82.2(76 .-7.0)$ \\
\hline IgA-TTG & $\begin{array}{l}30.6 \\
(18.7-45.6)\end{array}$ & $\begin{array}{l}91.6(83.6- \\
96.0)\end{array}$ & $\begin{array}{l}65.2 \\
(42.8-82.8)\end{array}$ & $\begin{array}{l}71.9 \\
(62.8-79.5)\end{array}$ \\
\hline IgA-EMA & $\begin{array}{l}34.3(26.7- \\
42.7)\end{array}$ & $\begin{array}{l}92.5(84.8- \\
96.7)\end{array}$ & $68.2(45.1-85.2)$ & $71.9(62.9-79.5)$ \\
\hline
\end{tabular}

\section{PTU-080 SHOULD WE ALL BE LOOKING FOR MARGINAL GAINS IN ENDOSCOPY EFFICIENCY?}

${ }^{1}$ Kathleen Bryce*, ${ }^{1}$ Cheh Kuan Tai, ${ }^{2}$ Sam Murray, ${ }^{1}$ Robert Fearn. ${ }^{1}$ Homerton University Hospital NHS Foundation Trust, London, UK; ${ }^{2}$ North Bristol NHS Trust, Bristol, UK

\subsection{6/gutjnl-2019-BSGAbstracts.439}

Introduction Demand for endoscopy in the UK has doubled in the last 5 years. In 2017, 64\% of units failed to meet suspected cancer targets despite $66 \%$ of units having weekend lists and $27 \%$ outsourcing to external providers ${ }^{1}$. UCL Cancer Collaborative (UCLCC) data showed demand can be met by improving efficiency. This is important in a resource-limited setting. Our Quality Improvement (QI) Project aimed to improve efficiency by improving turnaround time, non-attendance and on-the-day cancellations.

Methods The Endoscopy QI fellow, endoscopy unit manager and Gastroenterology service manager participated in the UCLCC Improvement Programme, and utilised QI methodology. We collected data from electronic patient records and scheduling system.

At baseline, we identified that underutilisation of lists was multifactorial. We introduced a turnaround nurse role to consent patients. Healthcare assistants (HCAS) and nurses were trained in cannulation. As poor bowel preparation contributed to cancellations, we introduced telephone pre-assessment to educate patients. Finally, the administrative team sent text reminders before appointments.

Results At baseline, our unit performed an average of 7.9 points per list, out of a planned 10. On average, 28.5 patients per month had procedures cancelled on the day due to poor bowel prep or inadequate fasting. After the introduction of pre-assessment, it improved to 23.5 per month, saving 5 procedures which would have had to be rearranged. The average points performed improved to 9.3 points per list.

The average DNA rate has improved from $9 \%$ to $7 \%$ after the introduction of text reminders. After the introduction of the turnaround nurse and HCA cannulation, turnaround time reduced from an average of 18 to 9 minutes between procedures. This could save 90 minutes over a 12-point list.

Despite these improvements, only $41.6 \%$ of lists are booked for 1-2 points. Inadequate staffing numbers and late start times are contributing factors. A start time audit showed that only 5 out of 27 lists in a week started within 10 minutes of supposed start times, a target for further cycles of this QI project.

Conclusions Multiple small improvements in efficiency can achieve significant impact on productivity. Interventions focused on turnaround time can reduce underuse of list time. Patient-centred approaches to procedural preparation may reduce squandered appointments. Sustainability of these improvements is difficult to assess in the short term but will be promoted by the continuing QI fellow role and implementation of endoscopy QI champions from the administrative and nursing team.

\section{REFERENCE}

1. Shenbagaraj, L., Thomas-Gibson, S., Stebbing, J., Broughton, R., Dron, M., Johnston, D. et al. (2018) Endoscopy in 2017: A national survey of practice in the UK, Frontline Gastroenterology, pp. 100970. DOI: 10.1136/flgastro-201-00970.

\section{PTU-081 ENHANCING EFFICIENCY IN ENDOSCOPY UNIT USING THE 'TIME AND MOTION' MODEL}

${ }^{1}$ Shivkumar Budihal ${ }^{*},{ }^{1} \mathrm{Ms}$ Isobel Esberger, ${ }^{1} \mathrm{Ms}$ Ann Fertwell, ${ }^{1} \mathrm{Mr}$ Richard Canetti, ${ }^{1,2}$ Adolfo Parra-Blanco, ${ }^{1,2}$ Krish Ragunath. 'Nottingham University Hospitals, Nottingham, UK; ${ }^{2}$ NIHR BRC at Nottingham University Hospitals and University of Nottingham, Nottingham, UK

\subsection{6/gutjnl-2019-BSGAbstracts.440}

Introduction Endoscopy Units throughout the UK are facing unprecedented pressures due to increasing demand with limited capacity. Our aim was to explore strategies to improve our endoscopy unit's efficiency. In order to identify targets for improvement we sought to undertake a 'Time and Motion' study in the endoscopy unit. Methods

The study was conducted at Nottingham City Hospital Endoscopy centre between 13th January 2017 to 10th February 2017. Procedures included were gastroscopy, colonoscopy, sigmoidoscopy, bronchoscopy, therapeutic endoscopy and endoscopic ultrasound. From the time a patient reports to the reception to the time discharge was made the patients journey was mapped using the 'time and motion' principle. As part of this study, healthcare professionals and admission staff were asked to record the patient's journey through endoscopy unit accurately and complete templates which were kept with the patient's notes as they moved through the unit. Separate 
independent observers were then responsible for observing and recording times at which patients entered and exited the procedure room coupled with noting any issues for delays.

Results A total of 509 day case patients and 27 inpatient procedures were performed in 4 procedure rooms during the study period over 138 endoscopy lists. The adjusted median delay in starting time was 26 minutes with a peak of 20 minutes. The adjusted median turnaround times (time period between exit of patient from endoscopy room to entry of next patient in to the room) was $13: 11$ minutes with a peak at 12:30 minutes. The median for overruns (overrunning of lists beyond schedule) was 49:00 minutes. The adjusted median procedure time was 25:00 minutes. 95\% of endoscopy lists started late and $80 \%$ of the lists finished late. Bottlenecks identified were (1) delays in booking, (2) delays in patient preparation including enema, (3) inpatient transportation from and to wards and (4) timely endoscope availability. These were addressed by changing patient arrival times, additional support workers to support admission and patient preparation, introduction of co-ordinator to monitor lists with empowerment to redeploy staffing, and the role of a 'runner' to liaise between decontamination and the procedure rooms.

Conclusion The 'time and motion' study is a useful tool to identify areas for improvement in patient flow and improve efficiency in the Endoscopy unit.

\section{PTU-082 DEVELOPMENT OF LOCAL ALCOHOL SERVICES - EXPERIENCE FROM AN ENGLISH RURAL DISTRICT GENERAL HOSPITAL}

Rosie Firth Burnside*, Evelyn Lim, Brian Ho, Piyush Singh, Brian Horgan, Albert H Davies, John Keating. University Hospitals of Morecambe Bay NHS Trust, Barrow-In-Furness, UK

\subsection{6/gutjnl-2019-BSGAbstracts.441}

Introduction Alcohol services are the cornerstone to delivering good alcohol detoxification treatment and outcomes for patients with alcohol dependence. The impact of staff educational program, e-CIWA proforma and inpatient outreach service for community rehabilitation (The Well) is assessed in this two-cycle audit study, leading to improvement in patient care and ongoing local service development at an English rural district general hospital.

Methods The 1st audit cycle was carried out between September 2016 and August 2017 at the Furness General Hospital in Cumbria. Adult patients admitted to Gastroenterology with alcohol related ICD-10 codings and an inpatient stay $>24$ hours were included in the study. A set proforma was used for data collection from the local electronic health record assessing standard of care against NICE recommendations. Outcomes assessed include patient engagement with inpatient and community services, discharge with detox medication and attainment of help in community. Quality deficiencies identified lead to introduction of hospital inpatient outreach service for community rehabilitation (The Well Community team) funded by substance misuse service Unity, electronic CIWA proforma and staff educational program in August 2017. A repeat audit cycle between September 2017 and August 2018 was conducted to assess impact of intervention. Chi-squared/ Fisher exact test was used for proportions testing with $\mathrm{p}<0.05$ considered as statistically significant.
Results Baseline demographics between 2016/17 audit $(n=73)$ and 2017/18 audit cycle $(n=86)$ are similar. Comparing the latter audit cycle to the form, there is an increase in Magnesium level testing (34.3 to $73.3 \%, \mathrm{p}<0.0001)$ and IV Pabrinex prescription ( 89 to $97.7 \%, \mathrm{p}<0.02$ ). However there is a reduction in CIWA assessment documentation $(56.4 \%$ to $43.6 \%, \mathrm{p}<0.0091)$ and oral Thiamine prescription $(97.3 \%$ to $76.7 \%, \mathrm{p}=0.0002)$ respectively. Attainment of help by patients after discharge in the community increased (11 to $38.4 \%$, $\mathrm{p}<0.0001)$. There is no change in frequency of motivational assessment, prescription of community detox medication on discharge or referral to community rehabilitation. However, total referrals received from all sources to Unity have increased by $20 \%$.

Conclusions Infrastructure development (The Well) and staff education leads to improvement in quality of alcohol detoxification care. Integration of inpatient community rehabilitation service remains a challenge in the rural setting and needs further promoting. Reduction in CIWA assessment suggests need for dedicated QI focus for e-CIWA use. Repeated audit cycles allow impact assessment of service development measure and further study on impact towards re-admission rates and cost benefit are needed.

\section{\begin{tabular}{|l|l}
\hline PTU-083 TIME OF DAY INFLUENCES COLONOSCOPY OUTCOME \\
\hline
\end{tabular} MEASURES}

Talia Caspi ${ }^{*}$, Harshil Patel, Pradeep Mundre. Bradford Royal Infirmary, Bradford, UK

\subsection{6/gutjnl-2019-BSGAbstracts.442}

Introduction Endoscopic surveillance and removal of adenomas significantly reduces the incidence and mortality of colorectal cancer. There is concern that time of day could affect colonoscopy outcomes due to endoscopist fatigue over the course of the day. This could lead to interval cancers, and ultimately increased mortality. However, there are few studies in this area, and they have yielded inconsistent results. The aim of this study was to investigate whether evening colonoscopy lists achieve the same outcomes as daytime lists.

Methods Colonoscopy data from patients on the symptomatic pathway was gathered retrospectively over a one year period. Data was gathered only from endoscopists doing both daytime and evening lists. Data collected included quality of bowel prep, caecal intubation rate, adenoma detection rate (ADR), and colorectal cancer detection. Colonoscopy outcome data was compared between daytime and evening lists. Data was analysed using the $\chi^{2}$ test, and Fishers exact test.

Results 1150 colonoscopies were included in the analysis, of which 845 were from daytime lists, and 305 were from evening lists. Time of day produced a near significant difference in ADR [23.2\% $(n=196)$ daytime vs $17.97 \%(n=55)$ evening, $\mathrm{p}=0.0581]$. Detection of colorectal cancer was significantly lower in evening lists $[2.13 \% \quad(\mathrm{n}=18)$ daytime vs $0.33 \%$ $(n=1)$ evening, $p<0.05]$. Caecal intubation rate was significantly lower during evening lists $[96.69 \%(n=817)$ daytime vs 94.43\% ( $\mathrm{n}=305)$ evening, $\mathrm{p}<0.05$ ].

Conclusions It has been shown that the time of day significantly affects some outcome measures of colonoscopies. Demand for colonoscopies in the UK is rising year on year, and evening lists help to meet this demand. However, it must 